\title{
LA PRISON DE KAMINA ET SES PROBLEMES
}

\author{
Par Denis BWANA MBAYO, Chef de Travaux à l'Université de Kamina, et Emilie \\ SANGA KABAMBA, Assistante à l'Institut supérieur des techniques médicales de \\ Kamina
}

\section{INTRODUCTION}

La prison est généralement considérée comme l'étalon qui permet de prélever le degré de protection et de jauger le niveau de respect qu'un Etat de droit voue à la personne humaine fût-elle dans sa condition de détenue. Car, en dehors de la privation de liberté et d'une certaine restriction des droits subséquente, le détenu reste un homme à part entière. Or, les droits de l'homme qui ont pour épicentre « la dignité de la personne humaine » ont un caractère d'ordre public induisant le caractère self executing qu'on leur reconnaît du fait de leur nature objective ${ }^{1}$. Celle-ci, en effet, est une essence inhérente aux droits édictés par les dispositions constitutionnelles dans la mesure où ces droits loin d'être un coefficient statutaire révocable ad nutum, ont plutôt un caractère d'immanence et de permanence du seul fait de leur attachement à la seule qualité humaine sans aucun égard aux écarts par rapport aux normes de référence juridiques (criminels), biologiques (handicapés), mentales (aliénés) ou sociales (marginaux) ${ }^{2}$.

Cette étude porte sur la prison de Kamina en ce qu'elle aurait de particulier et de spécifique en rapport avec les exigences de garantie et de respect des droits des détenus. Et, c'est à l'aune des instruments internationaux ratifiés par la R.D.C. ${ }^{3}$ et de l'actuelle Constitution de la République Démocratique du Congo ${ }^{4}$ qu'elle sera évaluée et c'est en leur regard que s'articulent nos suggestions.

A cet effet, nous avons mené une enquête socio-juridique auprès des détenus, qui s'est étalée sur huit mois ${ }^{5}$, quant au respect des droits reconnus à la personne détenue et à l'effectivité de la défense de ces droits.

Précisons tout de suite que par détenu, au sens de cette étude, il convient d'entendre toute personne ou groupe de personnes qui, à la suite d'une condamnation par un tribunal

1 SUDRE, F. cité par YAV KATSHUNG, J., Les droits successoraux des enfants en République Démocratique du Congo, Thèse de doctorat en Droit, Université de Lubumbashi, 2006-2007, p. 254, inédit.

2 DELMAS-MALTY, M., « Le crime contre l'humanité, les droits de l'homme, et l'irréductible humain » in Revue des sciences criminologiques, $\mathrm{n}^{0} 3$, juillet-septembre 1994, p. 487.

3 Notamment Le Pacte international relatif aux droits civils et politiques, ratifié par la République Démocratique du Congo, le $1^{\mathrm{er}}$ novembre 1976 et publié au Journal Officiel, numéro spécial du 5 décembre 2002; Les Règles minima des Nations Unies relatives aux droits des personnes détenues.

4 Constitution de la République Démocratique du Congo publiée au Journal Officiel, numéro spécial, $47^{\mathrm{è}}$ année, Kinshasa, le 18 février 2006, telle que modifiée à ce jour.

5 Cette enquête a été menée du mois de septembre 2009 au mois de mai 2010. 
compétent ou d'une mesure conservatoire prise par un officier du ministère public ou un officier de police judiciaire, sont privées de leur liberté physique (d'aller et de venir) pour autant qu'elles soient placées dans un établissement pénitentiaire, dans une maison d'arrêt ou dans un amigo (cachot). Comme on peut bien le constater, notre champ visuel englobe donc même la personne arrêtée par un officier de police judiciaire et mise dans un amigo, alors qu'au sens du droit pénal, le détenu est une personne privée de sa liberté physique par décision de justice, soit au titre de la détention préventive ou provisoire, soit après avoir été reconnu coupable d'une infraction ${ }^{6}$. Au sens technique, le détenu ne doit pas être confondu avec l'individu placé en garde à vue ou sous contrôle judiciaire ${ }^{7}$.

\section{BREVE PRESENTATION DE LA BOURGADE DE KAMINA ET DE SA PRISON}

\section{A. La cité de Kamina}

\section{Données physiques}

Située au Sud-est du pays dans la province du Katanga, entre les latitudes $8^{0} 43^{\prime}$ et $8^{0} 46^{\prime}$ Sud et les longitudes $24^{0} 58^{\prime}$ et $25^{\circ} 02^{\prime}$ Est, la cité de Kamina est bâtie sur un plateau situé à une altitude voisine de 1100 mètres. Entité Administrative du territoire de Kamina, elle est l'émanation de la fusion des anciens territoires de Mato et Kinda. Originairement, Kamina relevait d'abord du District de Kabinda, ensuite de celui de Lulua et enfin de celui de Panda (Likasi alors Jadoville) avant d'être reconnu comme District autonome par l'Ordonnanceloi $\mathrm{n}^{0} 21 / 235 \mathrm{du} 4$ juillet $1952^{8}$. Il y a donc soixante-trois ans. Sa population s'évalue à environ 180.000 habitants $^{9}$ sur une superficie estimée à $6.060 \mathrm{~km}^{2}$.

\section{Données administratives}

La cité de Kamina, faut-il le rappeler, est une entité administrative urbano-rurale constituée d'une dizaine de quartiers et de deux villages (Kamina et Sobongo). Elle comprend en son sein trois camps dont deux pour la Police Nationale Congolaise : Camp Athénée et Camp

6 Le droit pénal congolais ignore la répartition tripartite qui tient compte de la gravité de la répression en distinguant les contraventions des délits et des crimes. Néanmoins, Il s'y réfère indirectement en matière de prescription de l'action publique. En effet, le législateur congolais fixe les délais légaux à un an révolu pour les infractions punissables d'un maximum de la servitude pénale ne dépassant pas une année ou d'une amende seulement; à trois ans révolus pour les infractions dont le maximum de la servitude pénale ne dépasse pas cinq années et enfin à dix ans révolus pour les infractions punissables de plus de cinq ans ou de la peine de mort (cfr. Article 24 du Code pénal congolais, Livre premier).

7 Cornu, G., Vocabulaire juridique, 7è édition, Paris, PUF, 2005, p.300.

8 C.T.B. PAIDECO KAMINA, Monographie de la Cité de Kamina, Kamina, juillet 2009, p. 13.

9 Ministère de l'Intérieur, Décentralisation et Sécurité du Katanga, District du Haut-Lomami. Rapport annuel, exercice 2006, Territoire de Kamina. 
Prison; et un pour la Police Militaire : Camp Tabora. La Base militaire est située à plus ou moins $25 \mathrm{Km}$ de la Cité de Kamina.

Kamina devrait avoir obtenu le statut de ville et par conséquent être devenue une Entité Territoriale Décentralisée au sens de la Loi sur la Décentralisation.

Le Gouverneur Belge du Katanga prit un Arrêté n ${ }^{0}$ 42/85/TG du 6 juin 1956 qui instituait la Cité de Kamina en un Centre extra coutumier. Avec le Décret- loi $n^{0} 081$ du 3 juillet 1998 portant organisation territoriale et administrative de la République Démocratique du Congo, ce Centre extra coutumier devenait une Cité subdivisée en une dizaine de quartiers, entités non autonomes dépendant de l'autorité de l'Administrateur de Territoire de Kamina par l'entremise du Chef de Cité.

Aujourd'hui, la Cité est en effet subdivisée administrativement en dix quartiers ${ }^{10}$ : le quartier Centre Urbain (28.388 hab.), le quartier $14^{11}$ (17.790 hab.); le quartier 52 (24.050 hab.); le quartier 53 (15.251 hab.); le quartier 64 (18.049 hab.); le quartier 82 (18.972 hab.); le quartier Katuba I (21.723 hab.); le quartier Katuba II (23.800 hab.); le quartier Congo (10.181 hab.) et le quartier Bloc II Kaloba (3.887 hab.).

L'adoption par le parlement de la nouvelle loi sur le découpage territorial donne à Kamina, Chef-lieu du District du Haut-Lomami, la vocation de Chef-lieu de la future province du Haut-Lomami conformément à l'article 2 alinéa $2^{0}$ de la Constitution.

\section{B. La Prison de Kamina}

Le défaut d'archives n'a pu permettre de situer avec exactitude dans le temps, l'instauration de la prison du Haut-Lomami à Kamina. Tout ce que l'on sait c'est que vers les années 1930 avant d'être converti en centre pénitentiaire, le bâtiment qui abrite aujourd'hui la prison servait d'entrepôt à l'actuelle Société Nationale des Chemins de fer du Congo (SNCC). Ce bâtiment vétuste, aux murs lézardés mesure $24 \mathrm{~m}$ de longueur et 17 de largeur, soit une superficie globale de 408 mètres carrés. Il comprend trois bureaux administratifs (la Direction, le Greffe et le Secrétariat), un local servant de dispensaire, un cachot pour les détenus trop bouillants, trois magasins pour la garde du matériel et outils de travail, des installations hygiéniques, sept loges ou cellules servant de dortoirs aux détenus et une cour intérieure. Une annexe a été construite en 2008 pour y loger des femmes détenues qui, avant, tout en ayant une cellule à part, avaient en partage la même cour intérieure avec des hommes. C'est en 2010 que la cellule pour femmes a été effectivement inaugurée.

10 C.T.B., PAIDECO KAMINA, Plan de Développement urbain Ville de Kamina, Kamina, août 2010, pp. 26-27.

11 Ces quartiers portent le nom relativement à leur année de création. 


\section{Conditions de détention}

Sous ce point, il sera successivement passé en revue la capacité d'accueil, l'encellulement ou le régime pénitentiaire, le logement et l'hygiène.

\section{a. Capacité d'accueil}

D'entrée de jeu, relevons que contrairement à ce que l'ordonnance $\mathrm{n}^{\circ} 344$ du 17 septembre 1965 en son article 5 recommande, il n'y a pas de maison d'arrêt attachée à la prison de Kamina. Détenus, condamnés, hébergés et ceux qui bénéficient des mesures de sûreté, tous sont admis au même titre que les condamnés dans les mêmes cellules et subissent le même régime disciplinaire. Les cellules sont désignées et identifiées par les noms des Etats et Villes certainement en raison de leur charge symbolique ou de leur densité allusive : BELGIQUE, CHICAGO ${ }^{12}$, CHINE $^{13}$, ETHIOPIE, IRAK, SOWETO et URSS...

A la date du 10 février 2015, la population carcérale par cellule est ainsi répartie:

\begin{tabular}{|l|l|l|l|l|l|l|l|}
\hline Cellules & BELGIQUE & URSS & CHINE & SOWETO & IRAK & ETHIOPIE & CHICAGO \\
\hline Effectifs & 39 & 34 & 04 & 41 & 31 & 28 & 04 \\
\hline Superficies & $41,5 \mathrm{~m}^{2}$ & $28 \mathrm{~m}^{2}$ & $24 \mathrm{~m}^{2}$ & $41,5 \mathrm{~m}^{2}$ & $31 \mathrm{~m}^{2}$ & $21,8 \mathrm{~m}^{2}$ & $71,5 \mathrm{~m}^{2}$ \\
\hline
\end{tabular}

b. Le système pénitentiaire.

La prison de Kamina organise un système qui veut que le condamné exécute sa peine en milieu fermé pour mieux traduire le sens de la privation de la liberté avec toutes les conséquences que cela emporte. Nous estimons que sous l'influence des conceptions nouvelles sur le but et le sens de la peine ainsi que sur le nécessaire respect de la dignité humaine, cette privation de la liberté devrait être moins utilisée comme une punition que comme un moyen de réadaptation sociale du condamné. Nous sommes loin de cette conception.

En effet, le condamné en plus de la souffrance qui résulte de la privation de sa liberté, est astreint aux règles qui assurent l'ordre et la sécurité dans l'établissement pénitentiaire en même temps qu'il est soumis à un régime disciplinaire ferme et rigoureux. Les prisonniers passent toute la journée assis à même le sol dans la cour intérieure ${ }^{14}$ qui, il faut le rappeler, n'excède pas $40 \mathrm{~m}^{2}$. La position accroupie est rigoureusement exigée quand on s'adresse au préposé ou à d'autres visiteurs venus en qualité d'avocat-conseil, de magistrat instructeur, ou encore de médecin traitant, pour éviter des évasions, pense-t-on. Le prisonnier ne peut donc ni parler débout, ni s'asseoir sur une chaise. Ce rare privilège est réservé aux capitas,

12 Cellule qui a été construite par la MONUSCO en 2008 et inaugurée en 2010 pour les femmes détenues.

13 Cette cellule héberge des gens d'une certaine classe sociale, des responsables pour la plupart.

14 La cour intérieure pour femmes a $32 \mathrm{~m}^{2}$ de superficie. 
différents responsables institués pour les besoins de la cause : discipline, mesures d'hygiène et de salubrité, régulation des entrées et des sorties, admission de nourriture venue de la famille ou autre correspondance destinée aux détenus.

Dans la cour comme en cellules, le silence est de rigueur pour minimiser les risques de concertations en vue d'un quelconque projet d'évasion ou d'agression des responsables. Les nouveaux venus sont souvent accueillis par des brimades et autres conditionnements éprouvants qui les obligent parfois à payer une certaine « rançon » pour avoir le bénéfice d'un traitement de faveur face aux nouvelles conditions de vie rendues insupportables à dessein par certains capitas.

Ni poste de radio, ni poste téléviseur, la réclusion coupe le détenu du monde. Néanmoins trois jours des visites sont prévus par semaine ${ }^{15}$. Les entretiens se passent dans un parloir commun sous haute surveillance d'un responsable qui est en mesure d'écouter les conversations. En tout cas, l'atmosphère y est, à tout le moins, débilitante pour ne laisser aucun espace susceptible de donner libre cours aux sentiments intimes.

On peut lire sur la porte d'entrée de la prison pour hommes : «KOTA OKOLA », ce qui peut être traduit du Lingala au Français par « Entrez et vous en sortirez agrandi ». Ce brocard fait allusion à l'endurance physique dont tout celui qui y est admis doit faire montre. En effet, la discipline qui y est imposée renforce le caractère afflictif de la peine privative de liberté. Elle a un caractère systématiquement vexatoire en ce qu'elle vise à neutraliser le prisonnier; à briser sa personnalité ${ }^{16}$.

Le système qui est d'application est celui de l'emprisonnement en commun : les prisonniers, réserve faite cependant de la séparation des hommes et des femmes, vivent ensemble aussi bien le jour que la nuit. Les cellules sont des dortoirs qu'ils partagent pour le sommeil comme pour les repas. Ils font ensemble des travaux de champs ${ }^{17}$. Le régime n'est donc ni pennsylvanien, ni auburnien.

Quant à l'ensemble de la population carcérale ${ }^{18}$, le tableau ci-dessous distingue à la date du 9 février 2015, les catégories ci-après:

\begin{tabular}{|l|l|l|l|l|l|l|l|}
\hline \multicolumn{1}{|c|}{ Catégories } & \multirow{2}{*}{ Hommes } & \multirow{2}{*}{ Femmes } & \multicolumn{5}{|c|}{ Provenance } \\
\cline { 4 - 9 } & & & TGI & TMG & Tri. Paix & PGI & Audit. Mil. \\
\hline Condamnés & 25 & 01 & 05 & 13 & 08 & - & - \\
\hline Prévenus & $152^{*}$ & 03 & $90^{*}$ & 07 & 07 & $15^{*}$ & 17 \\
\hline Totaux & 177 & 04 & 95 & 20 & 15 & 15 & 17 \\
\hline
\end{tabular}

15 Hormis les jours ferries, il s'agit de tous les mercredis, vendredis et dimanches.

16 BOUlOC, B., Pénologie, $3^{\mathrm{e}}$ éd., Paris, Dalloz, 2005, pp. 183-184.

17 Un champ de maïs (1,5 ha) dans la cour extérieure de la prison et un champ de manioc (1 ha) à Lunge (12 Km de la prison).

18 A la date du 9 février 2015, la population carcérale globale s'estime à 181 détenus dont 4 femmes. 
- Parmi les 152 prévenus, 19 ont interjeté appel.

- Parmi les 90 prévenus en provenance du Tribunal de Grande Instance, on note la présence d'une femme.

- Il y a 15 prévenus du Parquet de Grande Instance dont une femme.

Pour deux raisons majeures, on note que les prévenus sont plus nombreux que les condamnés : le Tribunal de Grande Instance de Kamina ne siège plus depuis juin 2013, faute de juges; le président étant resté seul alors que les nouvelles affectations des juges n'ont jamais pris effet.

On déplore aussi la pratique qui consiste à arrêter préventivement pour n'importe quoi en attendant que le suspect verse la caution pour sa liberté définitivement provisoire. Le concours de ces situations expliquerait la supériorité numérique des détenus prévenus par rapport aux condamnés.

\section{Salubrité et conditions d'hygiène}

La CICR avait construit un puits d'eau en 2008 dans l'enceinte de la prison, ce qui a largement contribué à l'amélioration des conditions d'hygiène qu'il y a dix ans. Cette eau permet au nettoyage régulier des toilettes ${ }^{19}$.

L'on peut cependant déplorer le fait que l'espace vital réservé aux effets de chaque détenu et à son logement soit trop réduit. Pas d'espace libre dans les dortoirs où une couchette de fortune étalée au sol sert de lit pour deux voire trois personnes dans bon nombre des cellules.

Les détenus sont jetés en pâture aux punaises et poux. Les pensionnaires de la prison de Kamina pensent avec nostalgie à la belle époque où le CICR avait fourni des produits pour la destruction de ces bestioles ${ }^{20}$.

\section{CONSTITUTION ET GARANTIE DES DROITS DE LA PERSONNE ARRETEE EN $R D C$}

La problématique des droits de l'homme repose essentiellement sur la notion de « garantie » de leur jouissance par les individus. Dans une moindre mesure, la Constitution serait une garantie en ce que les droits qui y sont prescrits obligent tout le monde, fût-il moralement, à les respecter et pourraient ainsi servir de fondement à une action en justice au bénéfice de la victime même si, au Congo Démocratique, la pratique judiciaire n'offre pas encore ou mieux suffisamment des cas d'application par le juge national des dispositions des

19 Il y a, pour les hommes, sept toilettes et trois douches; et pour les femmes, une toilette et deux douches.

20 Ce fut en mai 2005. 
instruments internationaux relatifs aux droits de l'homme ${ }^{21}$. Ceci est d'autant plus vrai que le droit congolais de Droits de l'Homme est d'émanation internationale ${ }^{22}$.

Il en découle que les conventions internationales ratifiées par la RDC s'imposent à elle et ses citoyens sont fondés à en revendiquer tant le respect que le bénéfice, ces Traités étant d'application d'office sur son territoire national ${ }^{23}$.

Au surplus, le constituant, lui-même, n'a-t-il pas prescrit à tous les pouvoirs constitués et à toute personne le devoir de respect absolu des droits de l'homme et de toutes les libertés fondamentales constitutionnellement consacrés ${ }^{24}$ ? Comme pour pérenniser ces droits et les prémunir contre tout acte qui aurait un effet péremptoire à leur endroit, n'a-t-il pas au pouvoir constituant lui-même récusé la moindre faculté d'en envisager une révision constitutionnelle visant à en réduire le nombre ${ }^{25}$ et la portée en dépit des circonstances exceptionnelles décrétées ${ }^{26}$ ?

De toute évidence, du moins sur le plan purement formel, la Constitution de 2006 présente plus de garantie et plante un décor qui restitue au souverain primaire son rôle en même temps qu'elle érige des écueils pour contrer le retour de la dérive dictatoriale et son cortège de violations flagrantes des droits reconnus aux citoyens. Non seulement le constituant de 2006 a, en les intégrant dans le corps même de la Constitution et non seulement en y faisant allusion dans le préambule comme ce fut le cas des constitutions précédentes, donné une valeur constitutionnelle aux droits et libertés; il y a également introduit d'innombrables droits nouveaux ${ }^{27}$.

A la personne arrêtée ou détenue, le constituant du 18 février 2006 reconnaît un certain nombre de droits devant être respectés par ceux qui font appliquer la loi, représentant de L'Etat ou agissant sous les ordres et le commandement d'une autorité compétente. Parmi ces droits, il est loisible de citer en passant :

$1^{\circ}$ Le droit pour la personne arrêtée de ne pas être soumis à la torture ou à un traitement cruel, inhumain ou dégradant (article 16 alinéa 4). Cet interdit rentre dans le noyau irréductible, dans les droits « indérogeables » de l'Homme. Aussi, à son article 61 point 2,

21 YAV KATSHUNG, J., Op.cit. p.252.

22 L'article 216 de l'actuelle Constitution règle et tranche le problème du conflit des lois entre les Traités et la Constitution en faveur de la primauté et de la suprématie des premiers sur tout autre ordonnancement juridique national.

23 Cette disposition est l'une des fondamentales de tradition constitutionnelle récurrente depuis 1964.

24 Article 60 de la Constitution du 18 février 2006.

25 Article 220, alinéa $2^{0}$ de la même Constitution de 2006.

26 Article 61 de la Constitution précitée.

27 Parmi ces droits, on peut citer entre autres la parité homme-femme (article 14), la reconnaissance d'une opposition politique (article 8); le droit de manifestation pacifique et publique sans aucune autorisation préalable, politique ou administrative (article 26), le droit à une assistance judiciaire à tous les niveaux de la procédure pénale, y compris l'enquête policière et l'instruction pré juridictionnelle et devant les services de sécurité (article 19, alinéas 3 et 4), le droit et le devoir de désobéir et de résister à un ordre manifestement illégal émanant de l'autorité publique ou de son agent...(article 28.). 
le constituant a-t-il radicalisé cette prohibition en ce que même les circonstances exceptionnelles telles que l'état d'urgence ou de siège n'y pourraient déroger.

$2^{\circ}$ Le droit de tout détenu de bénéficier d'un traitement qui préserve sa vie, sa santé physique et mentale ainsi que sa dignité (article 18 alinéa 5). Ce faisant, le constituant revient sur la circulaire n 04/008/In/PGR/70 du 16 mai 1970 du Procureur général de la République qui interdit aux officiers de police judiciaire toute violence à l'égard des personnes arrêtées. Ils doivent se garder de traiter sans humanité les personnes arrêtées, de les soumettre pendant l'interrogatoire à des actes et procédés qui brisent leur résistance physique en vue d'arracher un éventuel aveu. La pratique du fouet, de la matraque ou des menottes en vue d'infliger à la personne arrêtée une douleur inutilement excessive, le fait de méconnaître leur humanité en les plaçant nus; de les obliger à se coucher à même le pavement mouillé à dessein ou dans un lieu malsain, souillé d'urines ou de matières fécales, tous ces actes corrosifs qui humilient et animalisent l'homme sont proscrits.

$3^{\circ}$ Le droit de n'être poursuivi, arrêté et détenu ni pour un fait d'autrui, ni pour une action ou une omission qui ne constitue pas une infraction à la loi au moment où elle a été commise et au moment des poursuites, ni non plus pour dettes (articles 17 alinéa $8^{\circ}$, alinéa $3^{\circ}$ et 61 alinéa $6^{\circ}$ ).

$4^{\circ}$ En écho de l'article 9 du Pacte international relatif aux droits civils et politiques, ratifié par notre pays, le constituant a, à l'article 18 alinéa $1^{\circ}$, reconnu à la personne arrêtée le droit d'être immédiatement informée des motifs de son arrestation et de toute accusation portée contre elle, et ce, dans la langue qu'elle comprend. Elle devra, sitôt arrêtée, être également immédiatement informée de ses droits comme le prescrit l'alinéa $2^{\circ}$ de la même disposition.

$5^{\circ}$ Il est reconnu à la personne en état d'arrestation le droit d'entrer immédiatement en contact avec les membres de sa famille ou avec son avocat-conseil (article 18 alinéa $3^{\circ}$ ). Pour préserver l'intimité des entretiens entre personnes arrêtées et membres de famille ou avocats, il est de bon droit que ces entretiens se fassent naturellement à portée de la vue, et non à portée de l'ouïe d'un agent de prison ou d'un officier de police judiciaire.

$6^{\circ}$ Le droit de n'être pas gardé à vue pendant plus de 48 heures; d'être à l'expiration de ce délai soit relâché soit mis à la disposition de l'autorité compétente, c'est-à-dire de l'officier du ministère public. Cette prescription ne connaît pas d'exception en dehors du cas de force majeure consistant notamment en l'impossibilité d'acheminer immédiatement la personne arrêtée au parquet en raison de la distance à parcourir.

$7^{\circ}$ L'article 19 , à son $4^{\circ}$ alinéa, reconnaît à toute personne le droit de se défendre ellemême ou de se faire assister d'un défenseur de son choix en précisant que ce, à tous les niveaux de la procédure pénale, y compris l'enquête policière et l'instruction pré juridictionnelle et qu'elle le peut même devant les services de sécurité. Ce droit, plus que les autres, il faut l'en informer lors de son audition et surtout lui fournir des facilités raisonnables de l'exercer. 


\section{RESULTATS DE L'ENQUETE SOCIO-JURIDIQUE MENEE A LA PRISON DE KAMINA EN RAPPORT AVEC LE RESPECT DES DROITS HUMAINS}

L'enquête socio juridique que nous avons menée à la prison du District du Haut-Lomami sur le respect des droits reconnus à la personne détenue et sur l'effectivité de la défense de ces droits s'est étalée sur une période de 8 mois.

Au cours de cette enquête, les effectifs de départ qui étaient de 179 détenus sont passés au 30 mai 2010 à 267 parmi lesquels 156 prévenus, 19 hébergés, 6 ayant interjeté appel, 1 vagabond et 2 mineurs (mesures de sûreté) et 79 condamnés à la servitude pénale principale.

Le questionnaire, outre la situation du détenu, comportait des questions portant sur la perception de la détention, l'état de lieux de la violence carcérale ainsi que la connaissance des droits du détenu et la dénonciation des abus subis en prison. Considérée a quo, la taille de l'échantillon (64 personnes interrogées) représente 35,8\% et ad quem $24 \%$. Les graphiques qui suivent sont produites par nous à la suite des résultats observés.

\section{Analyse de la représentativité de l'échantillon:}

En rapport avec le sexe :

- Hommes : $56(87,5 \%)$

- Femmes : $08(12,5 \%)$

Dix femmes au mois de septembre 2009 et vingt au mois de juin 2010 sur respectivement 179 et 267 détenus, les femmes sont minoritaires $(5,6 \%$ et $7,5 \%)$ : considérer $12,5 \%$ est largement représentatif pour la population féminine et $87,5 \%$ pour celle masculine.

En rapport avec l'âge :

- Moins de 18 ans : $1(1,6 \%)$

- Moins de 25 ans : $15(23,4 \%)$

- Entre 25 et 35 ans : $35(54,7 \%)$

- Plus de 35 ans : $13(20,3 \%)$

La jeunesse de 20 à 35 ans est majoritaire parmi les détenus, aussi cette frange de la population carcérale est-elle importante dans l'échantillon $(78,1 \%)$

En rapport avec le statut :

- Prévenus : $26(40,6 \%)$.

- Condamnés : $38(59,4 \%)$.

Ce sont généralement (du mois de septembre 2009 au mois de juin 2010) les prévenus qui sont les plus nombreux pour des raisons que nous avons évoquées plus loin. Mais dans l'échantillon, il y a plus de condamnés $(59,4 \%)$ que des prévenus $(40,6 \%)$, c'est pour des raisons évidentes que les seconds y sont à titre temporaire et n'ont pas une immanence durable pour connaître à fond les réalités de la prison.

Lors de nos entretiens avec les détenus prévenus, certains n'avaient que deux jours de détention. C'est pour accorder plus de fiabilité aux réponses recueillies que nous avons pri- 
vilégié la catégorie des condamnés. Cette réalité se reflète d'ailleurs sur le temps passé en prison :

- Moins d'une année : $27(42,2 \%)$

- Entre 1 et 3 ans : $35(54,7 \%)$

- Entre 3 et 5 ans : $02(03,1 \%)$

- Plus de 5 ans : $00(00,0 \%)$

a. II. Perception de la prison par les détenus.

Graphique $\mathrm{N}^{\circ} 1$. Comment avez-vous été accueilli en prison?

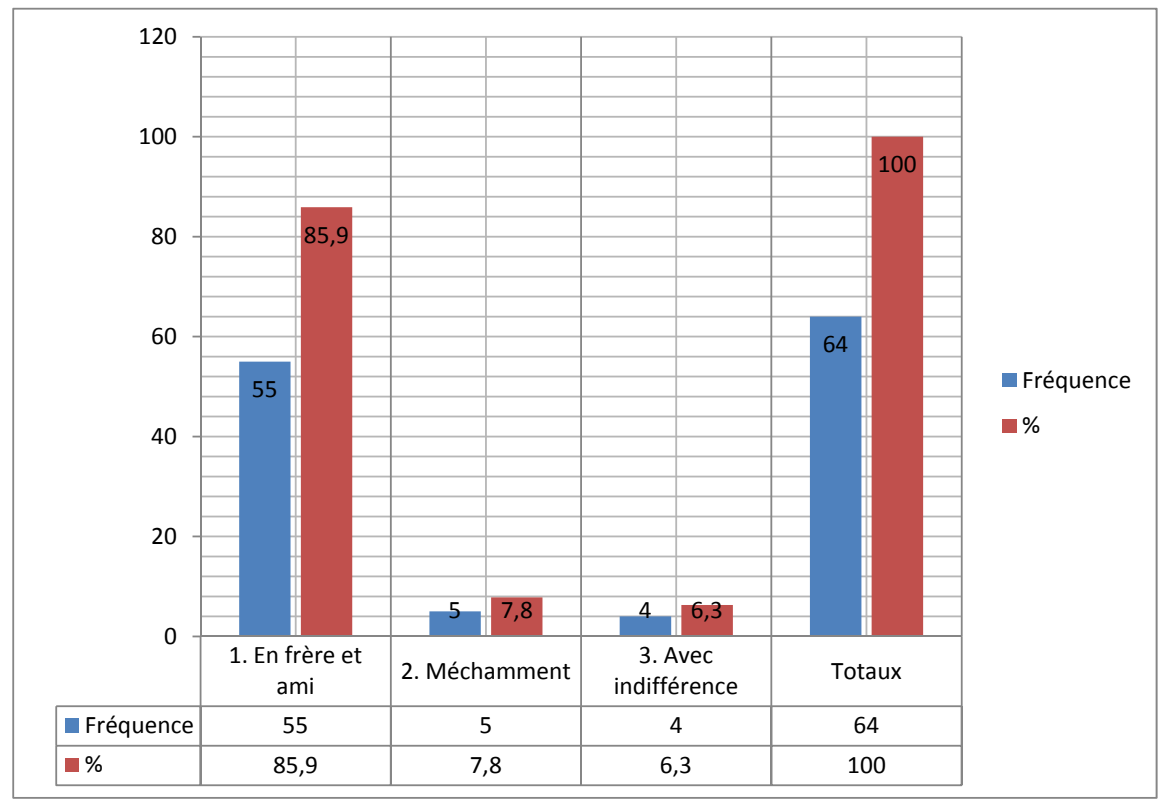

Il résulte des réponses recueillies qu'à l'accueil des nouveaux prisonniers, sous la surveillance des capitas, la rudesse à leur endroit s'est plus tempérée qu'il y a dix ans. 85,9\% des détenus reconnaissent avoir été l'objet d'un accueil fraternel et cordial en prison, tandis que la minorité dit avoir été reçue méchamment $(6,3 \%)$ ou avec une certaine indifférence $(6,3 \%)$. Ceci nous amène à dire qu'officiellement, les brimades à l'endroit des nouveaux venus ne sont pas permises, mais sont tolérées quelques rares fois. Nous estimons que seuls ceux qui ont une certaine responsabilité de fait parmi eux, peuvent de temps en temps se le permettre à l'endroit des autres. 
Graphique $\mathrm{N}^{\circ}$ 2. Comment trouvez-vous la vie en prison?

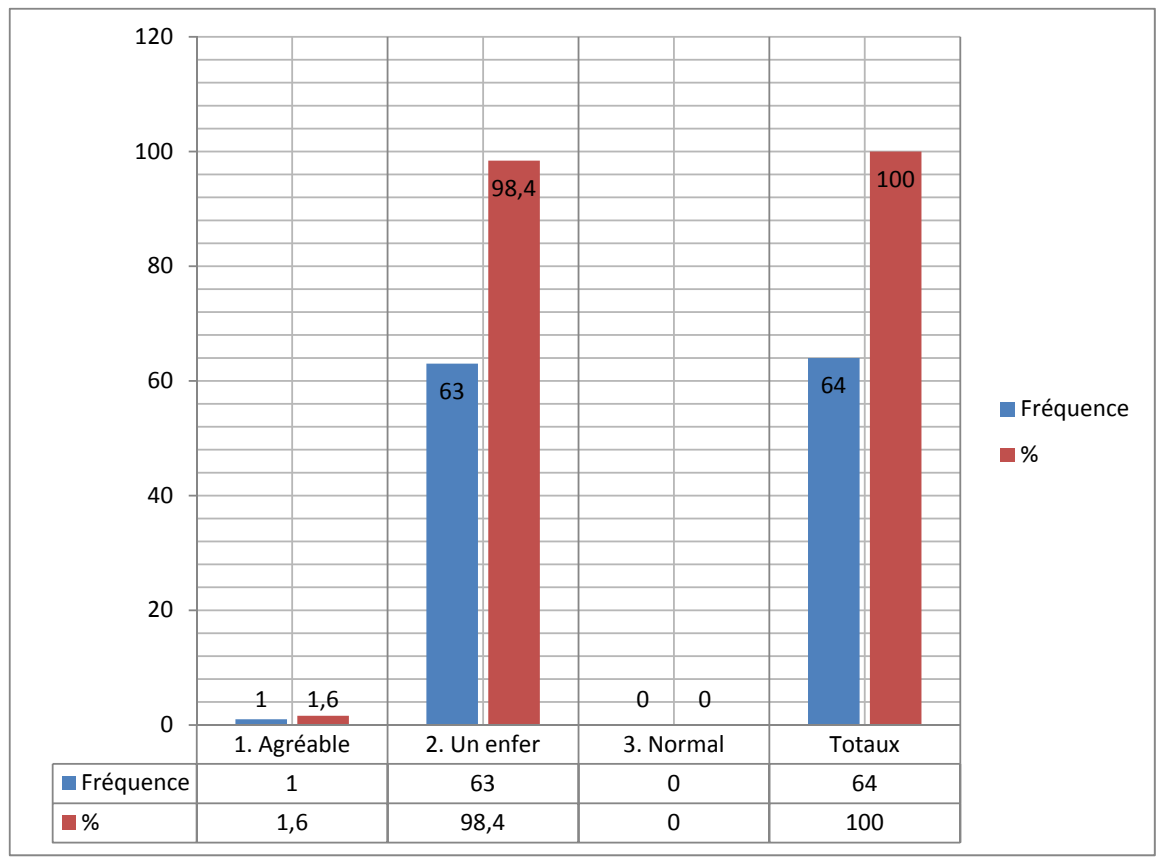

De ce tableau-graphique, il ressort que la quasi-totalité des détenus interrogés $(98,4 \%)$ trouvent que la vie de prisonnier est une vie difficile, pénible car la prison est par essence afflictive. On ne peut s'y plaire même si 1,6\% pense qu'il est agréable d'y être admis. En effet, les récidivistes n'ont plus rien à craindre de la détention. Il se trouve des gens qui, surtout parmi ceux qui y exercent une certaine fonction, préfèrent ne pas quitter la prison. Ils finissent par en faire un « état » et s'y faire. Ceux-ci sont plutôt rares. 
Graphique $\mathrm{N}^{\circ}$ 3. Qu'est-ce qui rend la vie de prison difficile?

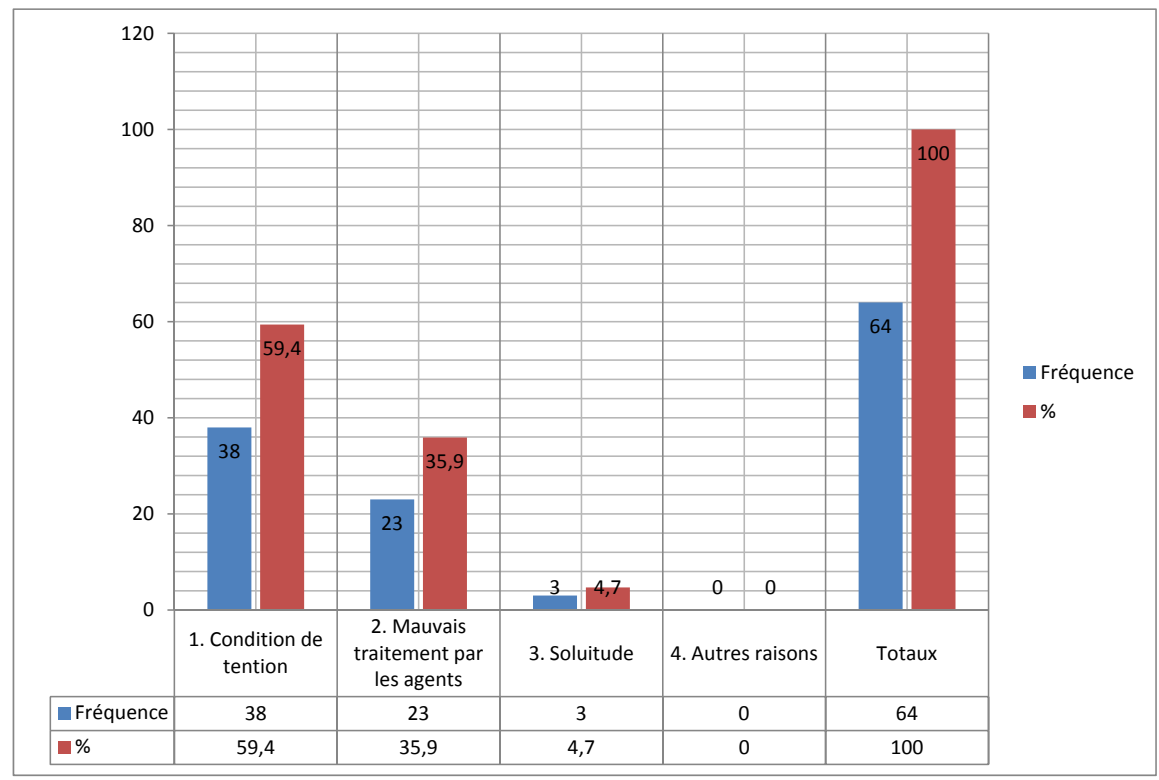

Ce tableau vient compléter et expliciter les réponses commentées au tableau précédent. Si presque à l'unanimité, les détenus sont d'avis que la détention a un caractère afflictif, la plupart d'entre eux $(59,4 \%)$, cependant, se l'expliquent par les mauvaises conditions de détention. La surpopulation, l'insuffisance de nourriture, l'infestation de poux et punaises, couche de fortune... sont autant de conditions qui rendent l'espace carcéral infernal, fantomatique.

D'autres enquêtés $(35,9 \%)$ accusent et déplorent le mauvais traitement qui caractérise les milieux carcéraux : ceux qui tiennent à leur dignité d'homme se sentent quelque peu chiffonnés, déshumanisés en raison du défaut d'humanité qui caractérise les détenteurs d'une « autorité de fait » sur eux d'une part et, les autorités pénitentiaires d'autre part. Dans tous les cas, on a souvent relevé la répugnance instinctive de l'administration à l'endroit de toute entrave apportée, même par la loi, à la conduite des affaires, sa répugnance à l'égard d'institutions susceptibles de critiquer, même sur des bases strictement objectives, « $s a$ » conception de l'intérêt public, «son » appréciation du fait et de l'adéquation de «sa » décision.

N'est-il pas vrai, comme le disait Montesquieu, que c'est une expérience éternelle, que tout homme qui a du pouvoir est porté à en abuser; qu'il va jusqu'à ce qu'il trouve des limites $^{28}$ ? 
Quant aux « autorités de fait » choisies parmi les détenus et qui veillent au grain, on relèvera de même qu'elles ne maintiennent pas facilement ni volontiers plusieurs objectifs contradictoires dans leur champ de visée. Si des limites rigides et parfaitement reconnaissables ne leur sont pas préalablement fixées et fréquemment rappelées, il est à craindre que ces " agents ", plutôt que d'assurer la défense rigide de solutions transactionnelles, ne cèdent au «monoïdéisme », aux sollicitations internes de leur rôle, de leur action et de leurs objectifs rapprochés. ${ }^{29}$

Enfin, des personnes interrogées, très peu $(4,7 \%)$ trouvent la prison insupportable du fait de la solitude psychologique et affective : la séparation d'avec leur milieu de vie normal les place dans une espèce d' " insularité » que rien ne peut consoler. Ce rare cas a été invoqué par des «mères » de famille qui caressent l'espoir de revoir un jour leurs enfants : l'instinct maternel.

Graphique $\mathrm{N}^{\circ} 4$. En quoi la prison vous effraie-t-elle?

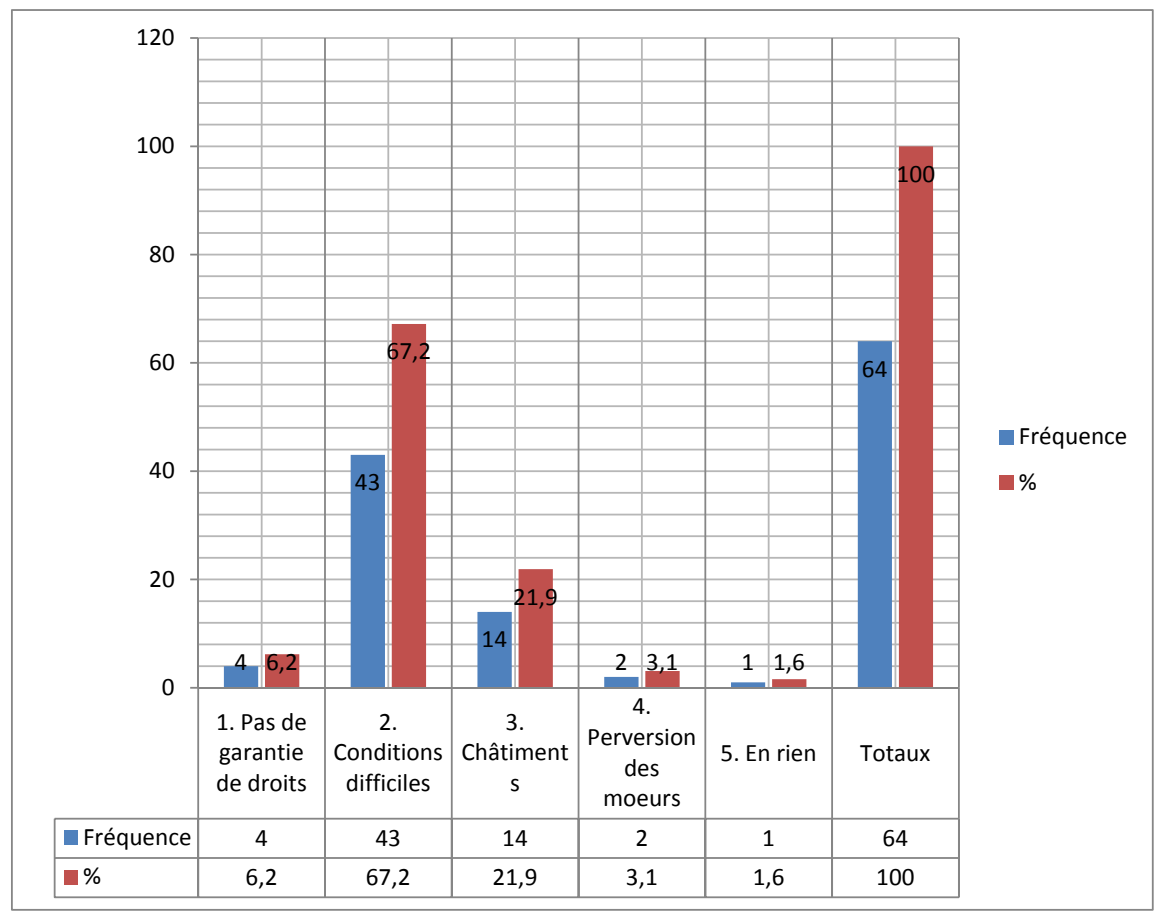

excès du pouvoir et la résistance légitime à l'autorité, Bruxelles, établissement Emile Bruyant, 1969, p. 68, note 31.

29 Ibidem. p.69. 
Il n'est que normal que ce qui rend la prison pénible puisse du même fait effrayer les détenus : les conditions difficiles de détention. Ce sentiment est ressenti et partagé par 67,2\% des détenus; viennent ensuite, conséquence logique de ce qui a été dit plus haut, les traitements inhumains, cause exprimée par $21,9 \%$ des détenus interrogés. Le reste de la population carcérale, craint soit la situation de non droit qui prévaut en prison $(6,2 \%)$, soit la corruption des mœurs par contagion $(3,1 \%)$ ou reste flegme $(1,6 \%)$. Ce dernier cas renvoie certainement à la catégorie de détenus qui se plaisent en prison (voir tableau $n^{\circ} 2$ ). $\mathrm{C}^{\prime}$ est une exception qui confirme la règle. La faible proportion (6,2\%) qui craint l'absence de garantie de droits s'explique par le fait que très peu sont informés des droits dont ils jouissent pour en craindre la violation et le manque de garantie quant à ce. Et une forte majorité est déjà dans la délinquance pour craindre une quelconque entame de l'attachement qu'ils vouent aux valeurs morales et aux normes sociales; c'est ce qui fait que seulement 3,1\% puissent se faire des soucis pour ce qu'ils seront devenus après leur séjour criminogène en prison.

\section{Etat de lieux des violences carcérales}

Graphique $N^{\circ}$ 5. Comment trouvez-vous le régime disciplinaire de la prison?

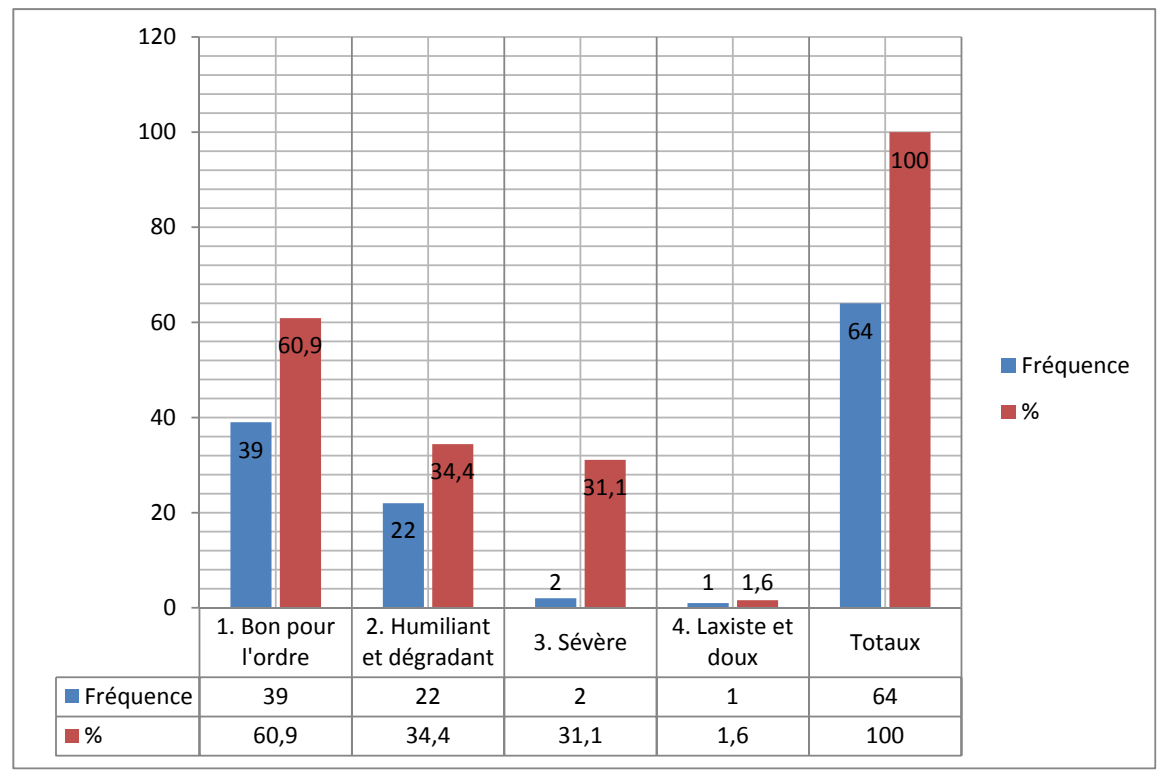

Il découle de ce tableau que $60,9 \%$ des détenus interrogés estiment que le régime disciplinaire instauré en prison est bon pour le maintien de l'ordre. Pour être vivable, c'est normal que la prison ne soit pas un milieu « anomique ». C'est un bon signe que la plupart d'entre la population cible ne soient pas réfractaires au caractère normatif de la « société » dans 
laquelle ils vivent. 34,4\% d'entre eux trouvent que le régime disciplinaire qui les régit ne tient pas compte de la dignité humaine bien qu'il soit nécessaire pour le maintien de l'ordre. Il faut concilier les deux fins qui ne sont pas nécessairement antinomiques; 3,1\% le trouvent plutôt sévère, tandis qu'une infime minorité, toujours la même, pense que le régime est doux (1,6\%).

Graphique $\mathrm{N}^{\circ} 6$. Avez-vous déjà été victime de violences physiques en prison?

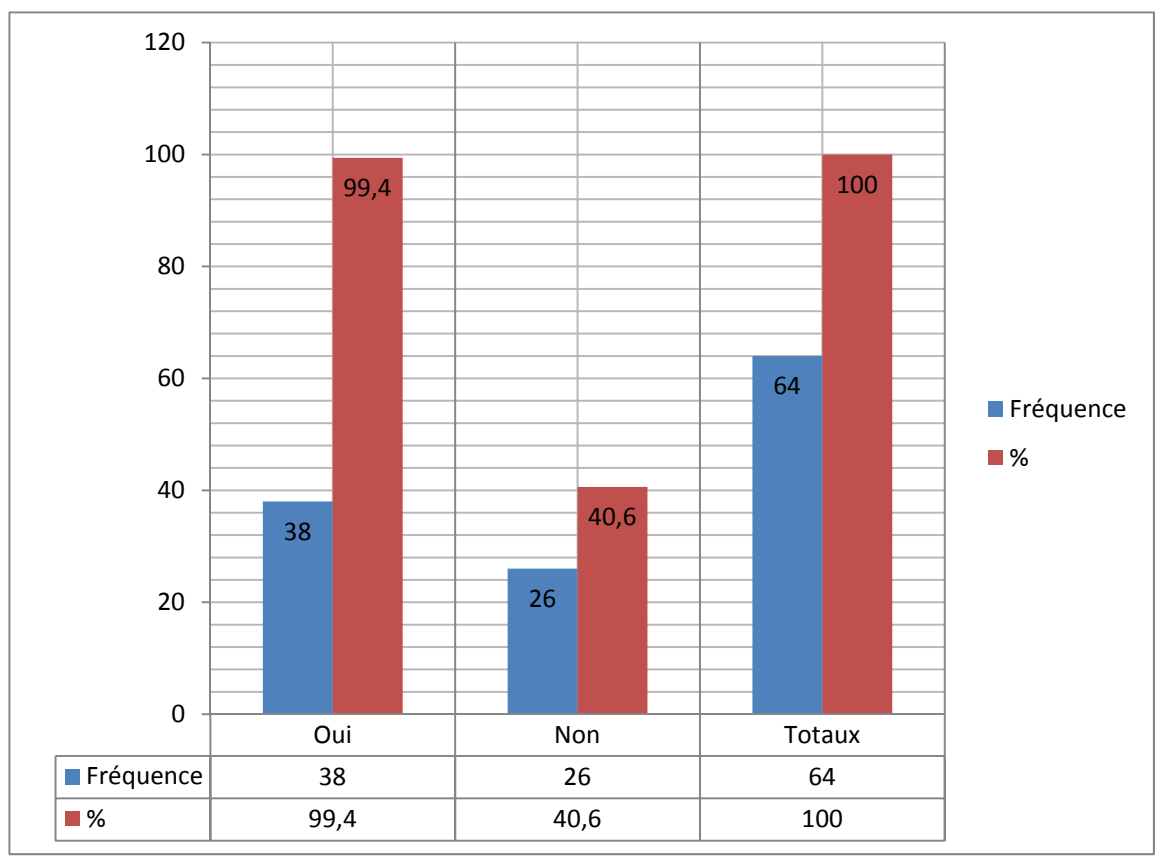

A la question de savoir si l'on a déjà été l'objet de violences physiques en prison, 59,4 \% ont répondu négativement et $40,6 \%$ ont répondu à l'affirmative. Le moins qu'on puisse dire c'est qu'il y a relativement peu de cas de violence, mais que la prison n'est pas exempte de violences. Ces résultats sont à relativiser dans la mesure où, pour une raison ou une autre, les détenus dans leur large majorité ont peur de dénoncer le mal qu'on leur a fait comme le montre le tableau ${ }^{\circ} 12$. Le nombre des victimes de violences carcérales pourrait donc être plus important que renseigné, outre le fait qu'il y en a parmi eux qui n'ont que quelques jours de détention. 
Graphique $N^{\circ} 7$. Avez-vous déjà été témoin des violences en prison?

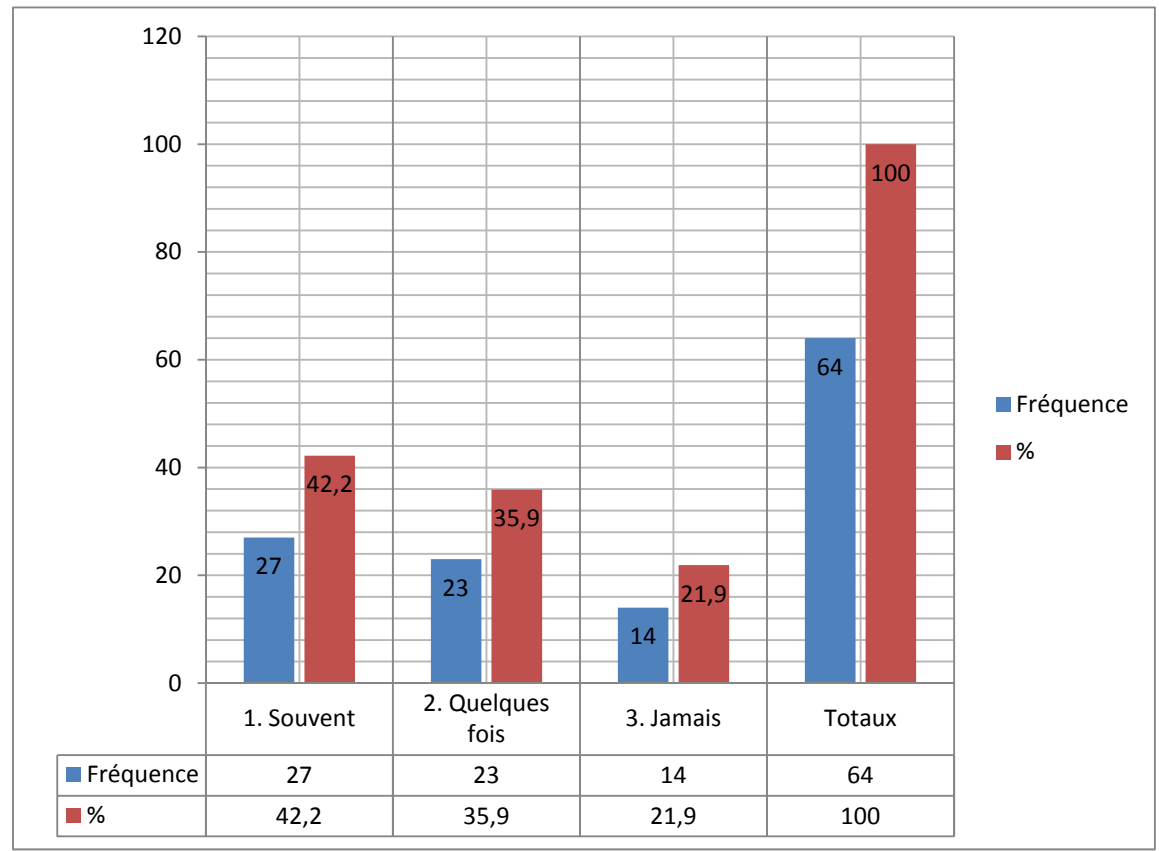

Les premiers témoins de violences, ce sont d'abord les victimes elles-mêmes. C'est ainsi que cette catégorie, à peu de chose près, dit qu'elle a «souvent » été témoin des violences en prison (42,2\%). Une autre grande partie de détenus dit avoir assisté quelques rares fois à la perpétration de la violence en milieu pénitentiaire $(35,9 \%)$, tandis que $21,9 \%$ des détenus disent n'avoir jamais été témoins des violences carcérales. Tout compte fait, ceux qui attestent le déploiement de la violence en milieu carcéral sont majoritaires (soit 78,1\%). Nous estimons que les nouveaux admis ayant moins d'une année représentent, il faut le rappeler, $42,2 \%$ des détenus interrogés. Ils sont à prendre en compte quant aux sujets qui disent n'avoir jamais vu ces violations. 
IV. Connaissance des droits et dénonciation de leur violation.

Graphique $N^{\circ}$. Savez-vous qu'en cas de mesures disciplinaires jugées injustes ou illégales à votre endroit, vous avez un droit de recours administratif et juridictionnel?

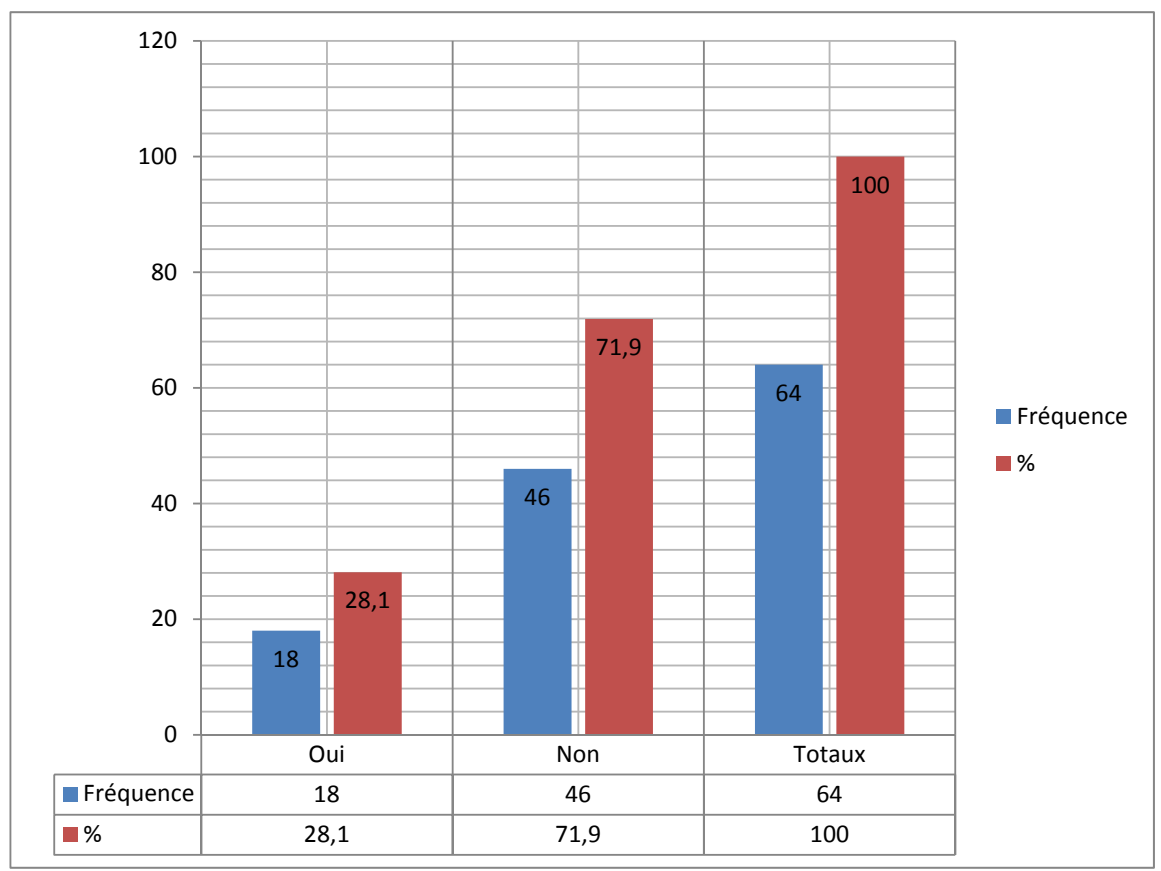

La plupart des détenus $(71,9 \%)$ ignorent la procédure administrative et la possibilité d'un recours pour excès de pouvoir devant une juridiction compétente. Le droit, pour la personne en détention, d'être informée de ses droits, n'a pas cours dans la prison de Kamina. Cet état de choses serait entretenu par ceux qui n'ont pas l'intention de respecter ces droits ou parce qu'eux-mêmes l'ignorent aussi.

$28,1 \%$ des détenus prétendent le savoir, connaissance infuse ou fruit d'un éventuel pré requis certainement. 
Graphique $\mathrm{N}^{\circ}$ 9. Savez-vous qu'en cas de violation flagrante de droits de l'homme dont vous seriez victime, vous pouvez vous en prévaloir en justice quand bien même vous seriez en état d'arrestation ou de détention?

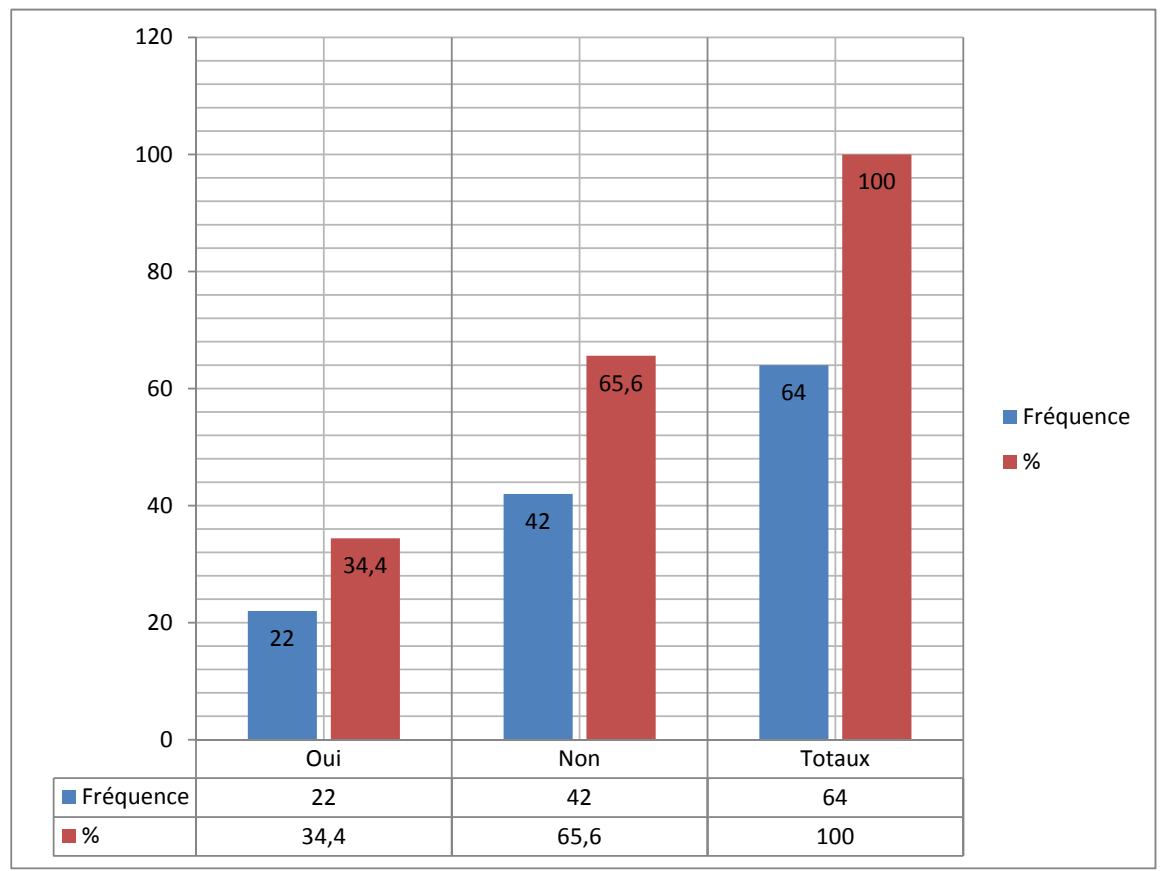

A peu de choses près, la réalité reste la même qu'il s'agisse de l'excès de pouvoir, de l'abus d'autorité ou de la violation des droits de l'homme : 65,6\% ignorent l'exercice du droit d'action en cas de violation des droits de l'homme et 34,4\% prétendent en être informés. Ce sont certainement les mêmes raisons qui expliquent cet état de choses. 
Graphique $\mathrm{N}^{\circ} 10$. Avez-vous déjà eu à dénoncer ces abus auprès d'un individu, de l'administration pénitentiaire ou d'un magistrat venu en visite?

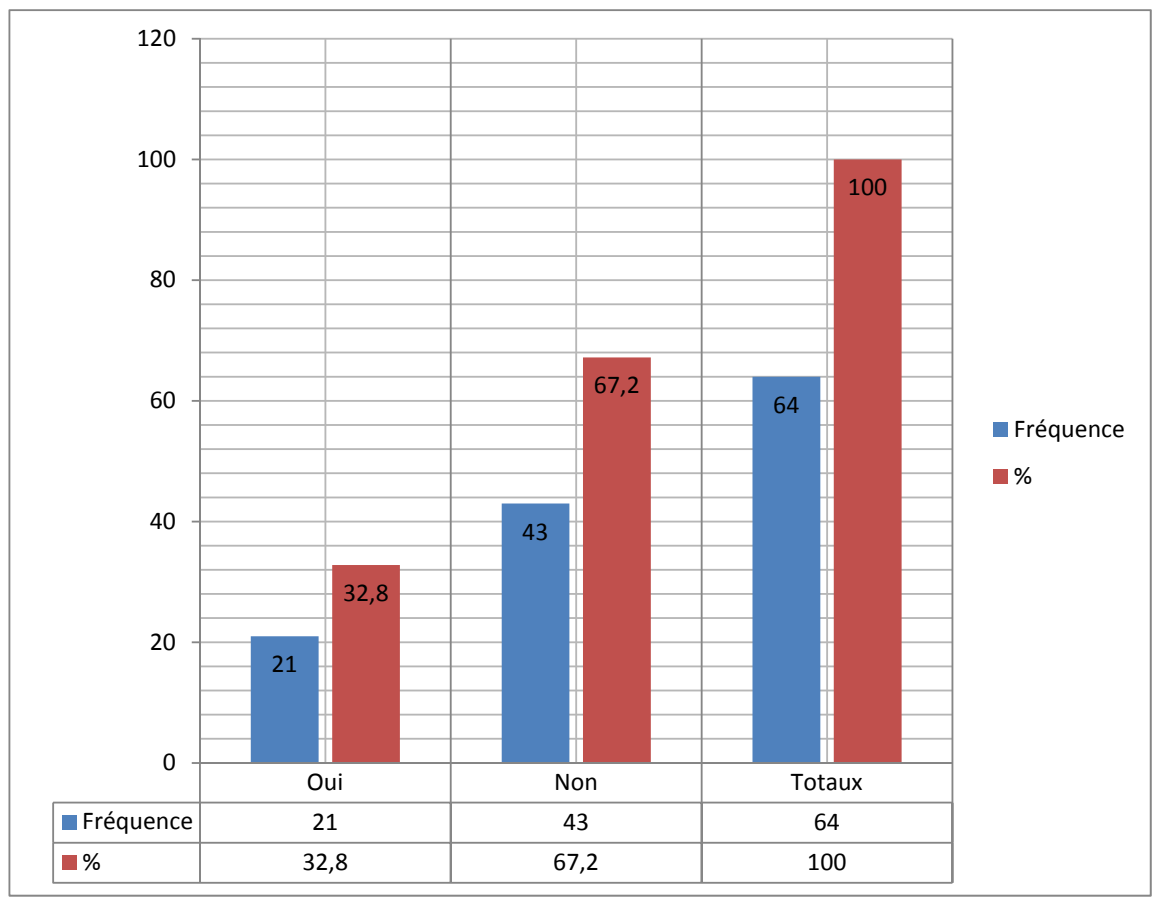

Toute proportion gardée, presque tous ceux qui prétendent connaître leurs droits, ont déjà osé en dénoncer le non respect par les autres $(32,8 \%)$ et ceux qui ignorent leurs droits ne peuvent, naturellement, pas les appeler à l'existence; et ils sont malheureusement les plus nombreux $(67,2 \%)$. 
Graphique $N^{\circ} 11$. Pourquoi n’avez-vous jamais dénoncé les cas de violation dont vous étiez témoin ou victime?

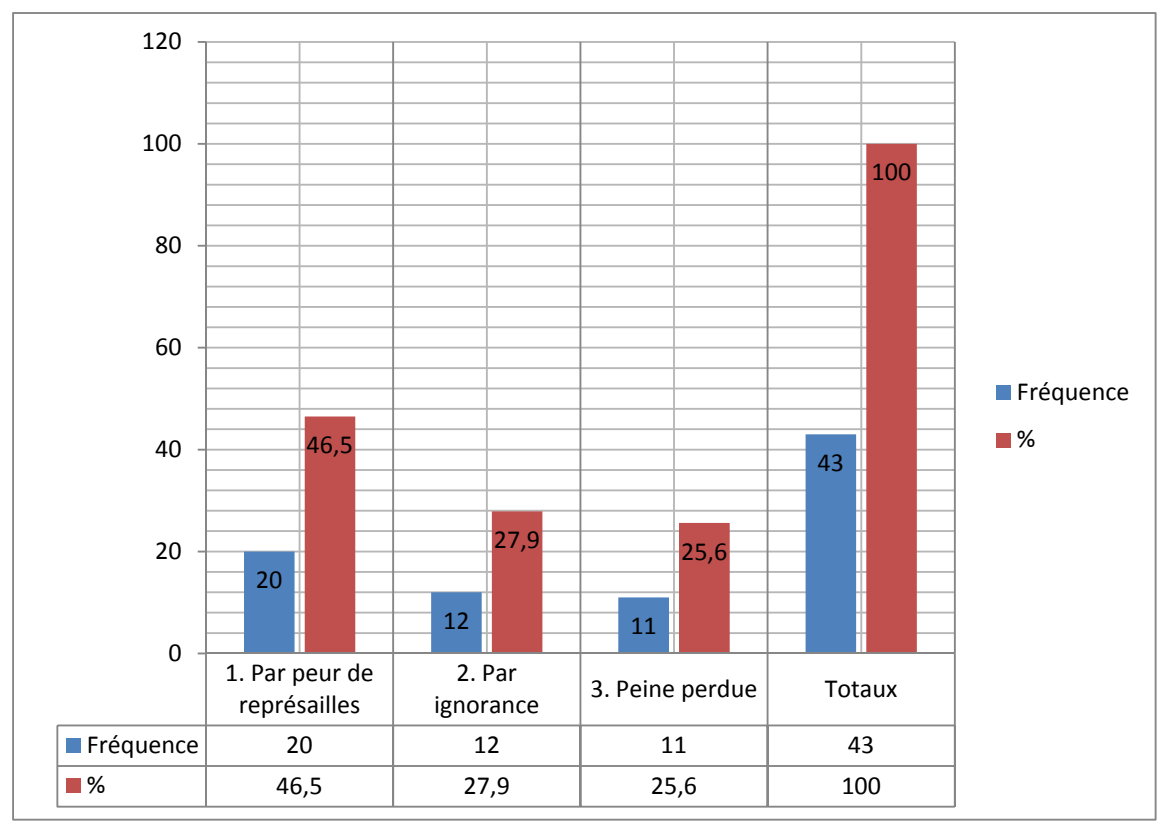

La tendance générale est de ne pas dénoncer les abus dont on est soi-même victime ou dont on est témoin. Parmi les raisons, la peur des représailles qui a été diversement exprimée par les personnes interrogées, est revenue plusieurs fois comme cause d'inhibition $(46,5 \%)$ : on est déjà en position de faiblesse et on s'attend à tout moment à bénéficier de la faveur de ceux qui exercent une certaine autorité sur soi; agir autrement serait aggraver sa situation et sa condition de détenu. Les autres pensent qu'une fois en état de détention, toute violence, tout abus dont on serait victime, est légitimé : cela ferait partie du lot quotidien du prisonnier qui serait de ce fait privé de tout droit (27,9\%); ou encore, il serait inutile de dénoncer les abus d'autorité et autres violations car les autorités jouissent d'une espèce d'immunité dans l'exercice de leur fonction qui consiste essentiellement en la puissance publique $(25,6 \%)$.

\section{CONCLUSION}

En définitive, la prison constitue en soi une violence, « une plaie » en ce qu'elle est afflictive en plus du fait qu'elle est le théâtre d'un traitement inhumain, non pas au moment de l'accueil des nouveaux admis, mais de façon informelle durant tout le séjour pénitentiaire. Les droits des détenus y sont pour une large part non respectés : conditions inhumaines de 
logement, insuffisance de repas, traitements dégradants, non information des droits reconnus aux prisonniers...

L'ignorance de droits, l'immunité administrative et la peur de dénoncer non seulement encouragent et favorisent la violation des droits réputés irréductibles, indélogeables, mais surtout « institutionnalisent » un déni d'action en prison rendant ainsi illusoire la garantie constitutionnelle dont ces droits sont couverts.

Cette enquête aboutit à des résultats, pour être de nature statistique, n'en sont pas moins des tendances, des lignes de sens qui traversent l'espace carcéral. Aussi, voudrions-nous aller au-delà de ces données pour lire l'arrière-fond des réponses recueillies auprès de ceux qui seraient tour à tour auteurs, victimes et témoins des violences carcérales.

L'expérience a démontré que pareilles études exigent impérativement un engagement affectif du chercheur envers le sujet de son étude et du temps nécessaire pour établir ce genre de liens avec les détenus ${ }^{30}$.

L'on comprend dès lors pourquoi nous avons mis neuf mois à fréquenter la prison. Cependant, il nous faut reconnaître que si le temps d'entrer en contact avec 64 détenus est celui que nous avons mis étant donné nos occupations, mais celui que nous devrions consacrer à chaque sujet n'était pas suffisant pour réaliser pleinement le vœu de De Greff et obtenir l'ouverture nécessaire à la révélation de la vérité.

Ceci est d'autant plus vrai que quand l'on croise les «tendances » qui résultent des réponses données, il sourd parfois une certaine contradiction en ce que le sujet peut nier ici ce qu'il reconnaît d'une façon ou d'une autre ailleurs. Un travail d'interprétation s'impose, audelà de l'apparente contradiction, pour comprendre les écarts, ce que cache le récit ou les réponses de l'auteur, de la victime ou du témoin des violences et autres violations de droits.

L'auteur craindrait sa propre condamnation alors qu'il s'attend, chaque jour qui passe, à une " providentielle libération ». Le détenu, auteur des violences, ne peut en aucun cas se dénoncer lui-même, il craindrait une réparation en faveur de sa victime. La victime, en dénonçant, pense, comme d'ailleurs le témoin, qu'elle sera inquiétée et menacée dans sa situation de détenu, par les codétenus ou les autorités défaillantes. Cette situation a des conséquences sur les résultats de l'enquête. On sent qu'il gît au fond des auteurs, des victimes ou des témoins privilégiés des violences, des sentiments négatifs de peur de dénoncer, de témoigner, peur d'être vrai et de dire la vérité. Dans le cas du détenu, invraisemblablement il y a un déni d'action qui s'installe par la force des choses. Et de ce fait, la garantie des droits du détenu se problématise.

Que l'Etat de droit entretienne avec la démocratie et le respect des droits humains des rapports de subsidiarité, c'est une évidence. En effet, l'Etat de droit est incontestablement et essentiellement lié à la limitation des pouvoirs des dirigeants par les règles de droit, afin d'éviter l'arbitraire et l'autoritarisme, et garantir ainsi la protection des droits humains. Mais, personne n'a la naïveté de croire que la séparation des principaux pouvoirs étatiques

30 CASONI, D. et BRRUNET, L., La psychocriminologie- Apports psychanalytiques et applications cliniques, Presses de l’Université de Montréal, 2003, p.61. 
et les droits de l'homme existent effectivement dans un Etat par le seul fait de leur affirmation constitutionnelle.

D'autre part, il n'est pas moins vrai que tout régime politique, même doté d'une Constitution écrite qui ne tiendrait pas compte déjà théoriquement d'attribuer à des organes distincts, dans les textes qui organisent la séparation des trois pouvoirs traditionnels, et à prévoir la constitutionnalisation et la protection des droits de la personne, ne peut prétendre être un régime constitutionnel. ${ }^{31}$

La constitutionnalisation des droits humains est une chose et le constitutionnalisme en est une autre. La consécration textuelle des droits et libertés reconnus aux citoyens et la culture du respect des textes et des valeurs constitutionnelles ne font pas toujours bon ménage en RDC. Cette culture fait cruellement défaut dans le chef de la majorité des dirigeants ${ }^{32}$.

Ces droits et libertés s'adressent avant tout à l'Etat et à ses représentants qui ne les proclament que parce qu'ils y ont été contraints par la force revendicatrice du peuple, le souverain primaire. Dès lors, il n'y aura de droits et libertés, même consacrés par la Constitution, que s'ils sont fondés par les citoyens qui en exigent et le respect et la reconnaissance effectifs par les pouvoirs publics. Il ne suffit donc pas de proclamer abondamment ces droits et libertés dans le corps d'une constitution pour estimer qu'ils sont garantis et en induire le règne d'un Etat de droit. Peu s'en faut.

\section{BIBLIOGRAPHIE SELECTIVE}

1. YAV KATSHUNG, J., Les droits successoraux des enfants en République Démocratique du Congo, Thèse de doctorat en Droit, Université de Lubumbashi, 2006-2007.

2. MALTY, M., « Le crime contre l'humanité, les droits de l'homme, et l'irréductible humain » in Revue des sciences criminologiques, $\mathrm{n}^{0} 3$, juillet-septembre 1994.

3. CORNU, G., Vocabulaire juridique, 7è édition, Paris, PUF, 2005.

4. BOUlOC, B., Pénologie, $3^{\mathrm{e}}$ éd., Paris, Dalloz, 2005.

5. VERHAEGEN, J., La protection pénale contre les excès du pouvoir et la résistancelégitime à l'autorité, Bruxelles, établissement Emile Bruyant, 1969.

6. CASONI, D. et BRRUNET, L., La psycho criminologie-Apports psychanalytiques et applications cliniques, Presses de l’Université de Montréal, 2003.

7. KAMUKUNY MUKINAY, Droit constitutionnel et Institutions politiques, Kinshasa, Editions Universitaires Africaines, Collection « Droit et Société », 2011.

31 C'est ce qu'en d'autres termes l'article 16 de la déclaration française des droits de l'homme et du citoyen voulait dire en déclarant : « toute société dans laquelle la garantie des droits n'est pas assurée ni la séparation des pouvoirs déterminée n'a point de constitution ».

32 En ce sens KAMUKUNY MUKINAY, Droit constitutionnel et Institutions politiques, Kinshasa, Editions Universitaires Africaines, Collection « Droit et Société », 2011, p. 327. 